\title{
Knowing your neighbours: How memory-mediated conspecific avoidance influences home ranges
}

\author{
Nathan Ranc ${ }^{1,2,3}$ (D) | Francesca Cagnacci ${ }^{2}$ (D) | Paul R. Moorcroft ${ }^{1}$ (D)
}

\author{
${ }^{1}$ Organismic and Evolutionary Biology, \\ Harvard University, Cambridge, MA, USA \\ ${ }^{2}$ Department of Biodiversity and Molecular \\ Ecology, Research and Innovation Centre, \\ Fondazione Edmund Mach, San Michele \\ all'Adige, Italy \\ ${ }^{3}$ Environmental Studies Department, \\ University of California Santa Cruz, Santa \\ Cruz, CA, USA \\ Correspondence \\ Nathan Ranc \\ Email: nathan.ranc@gmail.com
}

Handling Editor: Jennifer Gill

\begin{abstract}
In Focus: Ellison, N., Hatchwell, B. J., Biddiscombe, S. J., Napper, C. J., \& Potts, J. R. (2020). Mechanistic home range analysis reveals drivers of space use patterns for a non-territorial passerine. Journal of Animal Ecology. https://doi.org/10.1111/13652656.13292. Most animals for which space use has been studied restrict their movements into a constrained spatial area: their home range. The ubiquity of this space-use pattern suggests that home ranges are adaptive in a wide range of ecological contexts, and that they likely arise from general biological mechanisms. In this issue, Ellison et al. use a mechanistic home range analysis (MHRA) to uncover the drivers underlying home range patterns in a passerine that is non-territorial. They show that a model integrating both resource preferences (specifically, an attraction to woodland centre), and memory-mediated conspecific avoidance can capture the space-use patterns observed in a wild population of long-tailed tits Aegithalos caudatus. In doing so, their analysis extends the applicability of MHRA to capturing and predicting home range patterns beyond the previously studied cases where spatially exclusive home ranges emerge from scent mark-mediated avoidance responses to neighbouring groups.
\end{abstract}

\section{KEYWORDS}

Aegithalos caudatus, long-tailed tit, mechanistic home range analysis, movement, space-use
The movement of most animal species for which space-use has been studied is spatially constrained within a characteristic home range. The ubiquity of this space-use pattern suggests that: (a) site familiarity may be adaptive in a wide range of ecological systems, improving resource acquisition (Merkle et al., 2014; Spencer, 2012; Van Moorter et al., 2009) and/or reducing rates of mortality (Gehr et al., 2020); and (b) that home ranges likely result from general biological mechanisms (Börger et al., 2008). A variety of statistical models have been proposed to describe the spatial patterns of animal home ranges (Powell, 2000); however, these statistical estimators can only offer limited insights into the underlying drivers of spaceuse and how patterns of space-use might change in response to changes in the environment.

Mechanistic home range analysis (MHRA) develops predictions for patterns of animal space-use, by 'modelling the movement process' (Millspaugh \& Marzluff, 2001). This involves developing a mathematical formulation for the movement behaviour of individual animals: the fine-scale movement behaviour of each animal is described in terms of distributions of step lengths and directions of movement within successive time intervals, and how these distributions change in response to the animal's surrounding environment and/or interactions with other individuals. For example, animals often decrease the distance moved per unit time in areas of high food availability, or may avoid moving into areas where other individuals are present (e.g. Moorcroft et al., 2006). From this mathematical formulation of fine-scale individual movement behaviour, it is then possible to obtain equations for the predicted space-use of animals that result from them moving on a given landscape. Typically, these space-use equations take the form of an advection-diffusion based partial differential equations (PDEs) whose terms reflect the 
environmental and ecological factors affecting the animal's underlying movement behaviour.

To date, MHRAs have mostly been applied to mammalian carnivores using a cue-based formulation in which the combined influence of scent-mediated conspecific avoidance, and attraction towards a central-place (e.g. a den) can predict the formation of stable territories (Bateman et al., 2015; Briscoe et al., 2002; Giuggioli et al., 2011; Lewis \& Murray, 1993; Moorcroft \& Lewis, 2006; Moorcroft et al., 1999, 2006). In their recent article, Ellison et al. (2020) propose an alternate memory-based MHRA formulation, and show that it successfully captures the space-use patterns observed in a population of long-tailed tits Aegithalos caudatus (Figure 1a), a non-territorial passerine: the authors show that stable home ranges emerge from the combined influence of (a) memory-mediated conspecific avoidance, and (b) attraction towards the centre of woodland area. This is a significant contribution to the field of MHRA as it incorporates an alternate mechanism underlying the formation of home ranges, thereby extending the application of MHRA to non-territorial species. The MHRA formulation used in the analysis was first proposed in a theoretical paper by Potts and Lewis (2016), which demonstrated that, in the absence of both indirect territorial cues (e.g. scent marks) and a central-place (e.g. den site), constrained space-use can emerge from the memory-based avoidance of locations associated with previous conspecific interactions.

Ellison et al.'s (2020) analysis provides empirical support for this memory-based formulation of conspecific avoidance. Moreover, they formulate and test a suite of hypotheses pertaining to the influence of woodlands on long-tailed tit movements and resulting space-use patterns. Specifically, the authors explore whether the effect of tree cover on movement reflects taxis (i.e. influences directions of movement) or kinesis (i.e. alters the underlying distributions of movement speeds and turning angles), and whether these effects occur when individuals are located inside, outside or at woodland edges. Ellison et al. find that MHRA formulations that include advection towards woodland cores most accurately captured the long-tailed tits' home range patterns (Figure 1b,c). This formulation contrasts with previous MHRAs in which resources primarily influenced animal speed and turning angle (i.e. kinesis)-coyotes Canis latrans (Moorcroft et al., 2006) and meerkats Suricata suricatta (Bateman et al., 2015) travel more slowly in habitats that are rich in resources-but is similar to (taxis) avoidance of terrain features in the aforementioned studies. Close examination of the model's predictions reveals that observed long-tailed tit relocations are concentrated at the edges of the predicted home range, as opposed to the core of each home range (Figure 1c). This mis-match may be caused by the need to maintain regular presence or vocalize at home range edges to deter neighbouring flocks, or, alternatively, because long-tailed tits are responding to the finer spatio-temporal distribution of resources (e.g. ecotonal transitions between woodlands and pastures). An interesting avenue for further analysis of this dataset would be to explore these alternate behavioural hypotheses for the concentration of relocations at home range edges. One approach may be to formulate a model of fine-scale movement behaviour incorporating these effects of resources, such as redistribution kernels (Avgar et al., 2015; Ranc, Cagnacci, \& Moorcroft, 2020; Schlägel \& Lewis, 2014), and integrated resource selection analysis (Avgar et al., 2016), with the potential to advance our understanding of the mechanisms underlying resource selection by animals.

Ellison et al. (2020) show that the net effect of memory-mediated conspecific avoidance lead to the formation of exclusive home ranges, that is, little-to-no overlap between neighbouring long-tailed tit flocks. This pattern triggers many questions regarding the biological factors underlying such spatial segregation, and paves the way for future research on the continuum of space-use patterns between home ranges and territories. How important is cue-based conspecific avoidance versus memory-based avoidance? In this context, the spatio-temporal heterogeneity of food resources is likely to be important factor governing the exclusivity of space-use. For example, memory-based foraging can lead to spatial segregation between individuals in absence of conspecific avoidance or active resource defence (Riotte-Lambert et al., 2015). Separate biological mechanisms-conspecific avoidance (Ellison et al., 2020) and resource competition (Riotte-Lambert (a)

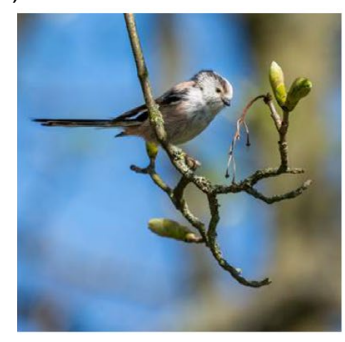

Long-tailed tit Aegithalos caudatus (b)

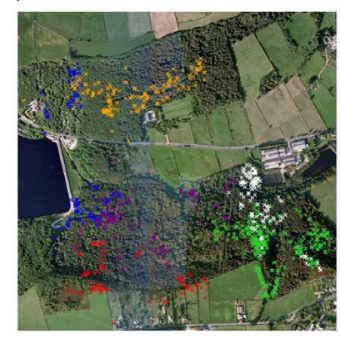

Observed flock relocations (c)

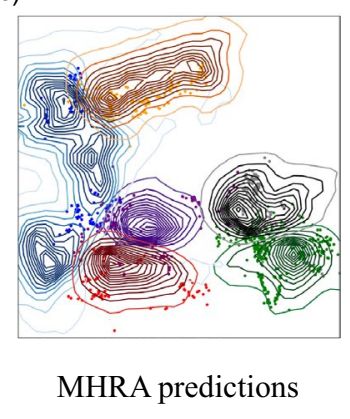

FIGURE 1 Ellison et al. (2020) developed a mechanistic home range analysis (MHRA) to uncover the biological drivers underlying space-use patterns in long-tailed tits Aegithalos caudatus (panel a). Panel (b) shows Ellison et al.'s relocations of six neighbouring flocks (dark green and light green/brown patches on the aerial image indicate woodland and agricultural areas, respectively). Panel (c) shows Ellison et al.'s space-use patterns for each flock as predicted by the best MHRA, which included memory-mediated avoidance of conspecifics and attraction to woodland centres 
et al., 2015)-may lead to the formation of exclusive, undefended home ranges. Identifying the respective influences of these two mechanisms in animal space-use patterns may prove challenging in observational studies. In this context, combining MHRA with resource manipulation experiments appears a promising avenue to disentangle these mechanisms (Fortier \& Tamarin, 1998; Jonsson et al., 2002; Krebs, 1971; Ranc, Moorcroft, Hansen, et al., 2020; Ranc, Moorcroft, Ossi, \& Cagnacci, 2020). A further avenue of research would disentangle the mechanisms of exclusive space-use from those promoting active home range defence, that is, the emergence of territoriality. This would require extensive work and necessarily include consideration of social and mating systems in the formulation of advection and attraction components of MHRAs.

Thanks to new emerging biologging techniques, researchers are now monitoring the movements of an increasing number of species, at ever finer spatio-temporal resolutions (Cagnacci et al., 2010; Kays et al., 2015). Methodological tools are being developed to take advantage of this wealth of data (e.g. Potts et al., 2018). Obtaining high-resolution movement datasets for a meaningful duration with respect to life span is often challenging when species are difficult to tag or are too small (Ossi et al., 2019). In this regard, Ellison et al.'s (2020) demonstration that MHRA is able to uncover the biological processes governing the space-use of long-tailed tits based on analysis of a modest-sized dataset of behavioural observations ( $n=981$ locations) is encouraging. Furthermore, the authors successfully test specific hypotheses pertaining to the role of relatedness and flock size in mediating conspecific avoidance (a given flock appears more likely to avoid larger and unrelated neighbouring flocks), showcasing the statistical power of MHRA even when the number of observations is relatively small. We agree with the authors that this conclusion would have been unlikely to be reached using traditional statistical home range estimators. In spite of the great potential of MHRA in providing an analytically tractable model linking animal movements and space-use that is amenable to predictions (e.g. Moorcroft et al., 2006; Moorcroft \& Lewis, 2006) and capable of uncovering complex behavioural drivers (Ellison et al., 2020), their use is still not widespread. Reducing the mathematical and computational challenges associated with formulating and fitting MHRA is required to make them accessible to a broader research community.

Theoretical analyses have shown that memory-based foraging (Bracis et al., 2015; Riotte-Lambert et al., 2015; Van Moorter et al., 2009), and memory-based avoidance of past interactions with conspecifics (Potts \& Lewis, 2016) can lead to the emergence of home ranges. In recent years, empirical evidence of the influence of memory on animal foraging decisions (Merkle et al., 2014; Ranc, Moorcroft, Ossi, \& Cagnacci, 2020), and ultimately space-use patterns (Merkle et al., 2017; Ranc, Cagnacci, \& Moorcroft, 2020) has been accumulating. Ellison et al. (2020) here provide the first empirical support for how spatial memory of conspecific interactions influences space-use patterns. Future studies should investigate whether similar memorybased formulations can be successfully applied to other species, and thus assess the generality of memory-based MHRAs to describe the formation and maintenance of animal home ranges.

\section{ACKNOWLEDGMENTS}

We thank N. Ellison and coauthors for sharing an illustration of their study species; we thank the editor, Jennifer Gill, for the valuable suggestions and feedback provided on an earlier version of this article.

\section{AUTHORS' CONTRIBUTIONS}

N.R. led the writing of the manuscript with support from P.R.M. and F.C. All authors contributed critically to the drafts and gave final approval for publication.

\section{ORCID}

Nathan Ranc iD https://orcid.org/0000-0002-3167-2251

Francesca Cagnacci (iD https://orcid.org/0000-0002-4954-9980

Paul R. Moorcroft iD https://orcid.org/0000-0002-2876-4673

\section{REFERENCES}

Avgar, T., Baker, J. A., Brown, G. S., Hagens, J. S., Kittle, A. M., Mallon, E. E., McGreer, M. T., Mosser, A., Newmaster, S. G., Patterson, B. R., Reid, D. E. B., Rodgers, A. R., Shuter, J., Street, G. M., Thompson, I., Turetsky, M. J., Wiebe, P. A., \& Fryxell, J. M. (2015). Space-use behaviour of woodland caribou based on a cognitive movement model. Journal of Animal Ecology, 84(4), 1059-1070. https://doi.org/10.1111/1365-2656.12357

Avgar, T., Potts, J. R., Lewis, M. A., \& Boyce, M. S. (2016). Integrated step selection analysis: Bridging the gap between resource selection and animal movement. Methods in Ecology and Evolution, 7(5), 619-630. https://doi.org/10.1111/2041-210X.12528

Bateman, A. W., Lewis, M. A., Gall, G., Manser, M. B., \& Clutton-Brock, T. H. (2015). Territoriality and home-range dynamics in meerkats, Suricata suricatta: A mechanistic modelling approach. Journal of Animal Ecology, 84(1), 260-271. https://doi.org/10.1111/1365-2656.12267

Börger, L., Dalziel, D. B., \& Fryxell, M. J. (2008). Are there general mechanisms of animal home range behaviour? A review and prospects for future research. Ecology Letters, 11(6), 637-650. https://doi. org/10.1111/j.1461-0248.2008.01182.x

Bracis, C., Gurarie, E., Van Moorter, B., \& Goodwin, R. A. (2015). Memory effects on movement behavior in animal foraging. PLoS One, 10(8), e0136057. https://doi.org/10.1371/journal.pone.0136057

Briscoe, B. K., Lewis, M. A., \& Parrish, S. E. (2002). Home range formation in wolves due to scent marking. Bulletin of Mathematical Biology, 64(2), 261-284. https://doi.org/10.1006/bulm.2001.0273

Cagnacci, F., Boitani, L., Powell, A. R., \& Boyce, S. M. (2010). Animal ecology meets GPS-based radiotelemetry: A perfect storm of opportunities and challenges. Philosophical Transactions of the Royal Society B: Biological Sciences, 365(1550), 2157-2162. https://doi.org/10.1098/rstb.2010.0107

Ellison, N., Hatchwell, B. J., Biddiscombe, S. J., Napper, C. J., \& Potts, J. R. (2020). Mechanistic home range analysis reveals drivers of space use patterns for a non-territorial passerine. Journal of Animal Ecology, 1-14. https://doi.org/10.1111/1365-2656.13292

Fortier, G. M., \& Tamarin, R. H. (1998). Movement of meadow voles in response to food and density manipulations: A test of the food-defense and pup-defense hypotheses. Journal of Mammalogy, 79(1), 337-345. https://doi.org/10.2307/1382870

Gehr, B., Bonnot, N. C., Heurich, M., Cagnacci, F., Ciuti, S., Hewison, A. J. M., Gaillard, J.-M., Ranc, N., Premier, J., Vogt, K., Hofer, E., Ryser, A., Vimercati, E., \& Keller, L. (2020). Stay home, stay safe - Site familiarity reduces predation risk in a large herbivore in two contrasting study sites. Journal of Animal Ecology, 89, 1329-1339. https://doi. org/10.1111/1365-2656.13202

Giuggioli, L., Potts, J. R., \& Harris, S. (2011). Animal interactions and the emergence of territoriality. PLoS Computational Biology, 7(3), 1-9. https://doi.org/10.1371/journal.pcbi.1002008 
Jonsson, P., Hartikainen, T., Koskela, E. S. A., \& Mappes, T. (2002). Determinants of reproductive success in voles: Space use in relation to food and litter size manipulation. Evolutionary Ecology, 16(5), 455467. https://doi.org/10.1023/A:1020854525220

Kays, R., Crofoot, M. C., Jetz, W., \& Wikelski, M. (2015). Terrestrial animal tracking as an eye on life and planet. Science, 348(6240), aaa2478. https://doi.org/10.1126/science.aaa2478

Krebs, J. R. (1971). Territory and breeding density in the great tit, Parus major L. Ecology, 52(1), 2-22. https://doi.org/10.2307/1934734

Lewis, M. A., \& Murray, J. D. (1993). Modelling territoriality and wolfdeer interactions. Nature, 366(6457), 738-740. https://doi.org/10. 1038/366738a0

Merkle, J. A., Fortin, D., \& Morales, J. M. (2014). A memory-based foraging tactic reveals an adaptive mechanism for restricted space use. Ecology Letters, 17, 924-931. https://doi.org/10.1111/ele.12294

Merkle, J. A., Potts, J. R., \& Fortin, D. (2017). Energy benefits and emergent space use patterns of an empirically parameterized model of memory-based patch selection. Oikos, 126(2), 185-196. https://doi. org/10.1111/oik.03356

Millspaugh, J. J., \& Marzluff, J. M. (2001). Radio tracking and animal populations. Academic Press.

Moorcroft, P. R., \& Lewis, M. A. (2006). Mechanistic home range analysis. Princeton University Press.

Moorcroft, P. R., Lewis, M. A., \& Crabtree, R. L. (1999). Home range analysis using a mechanistic home range model. Ecology, 80(5), 16561665. https://doi.org/10.1890/0012-9658(1999)080[1656:HRAUA $\mathrm{M}] 2.0 . \mathrm{CO} ; 2$

Moorcroft, P. R., Lewis, M. A., \& Crabtree, R. L. (2006). Mechanistic home range models capture spatial patterns and dynamics of coyote territories in Yellowstone. Proceedings of the Royal Society B: Biological Sciences, 273(1594), 1651-1659. https://doi.org/10.1098/rspb.2005.3439

Ossi, F., Urbano, F., \& Cagnacci, F. (2019). Biologging and remote-sensing of behavior. In J. Chun Choe (Ed.), Encyclopedia of animal behavior (2nd ed., Vol. 3, pp. 429-446). https://doi.org/10.1016/B978-0-12809633-8.90089-X

Potts, J. R., Börger, L., Scantlebury, D. M., Bennett, N. C., Alagaili, A., \& Wilson, R. P. (2018). Finding turning-points in ultra-high-resolution animal movement data. Methods in Ecology and Evolution, 9(10), 2091-2101. https://doi.org/10.1111/2041-210X.13056

Potts, J. R., \& Lewis, M. A. (2016). How memory of direct animal interactions can lead to territorial pattern formation. Journal of the
Royal Society Interface, 13(118), 20160059. https://doi.org/10.1098/ rsif.2016.0059

Powell, R. A. (2000). Animal home ranges and territories and home range estimators. In L. Boitani \& T. Fuller (Eds.), Research techniques in animal ecology: Controversies and consequences (pp. 65-110). Columbia University Press.

Ranc, N., Cagnacci, F., \& Moorcroft, P. R. (2020). Memory drives the formation of animal home ranges: Evidence from a reintroduction. BioRxiv. https://doi.org/10.1101/2020.07.30.229880

Ranc, N., Moorcroft, P. R., Hansen, K. W., Ossi, F., Sforna, T., Ferraro, E., Brugnoli, A., \& Cagnacci, F. (2020). Preference and familiarity mediate spatial responses of a large herbivore to experimental manipulation of resource availability. Scientific Reports, 10. https://doi. org/10.1038/s41598-020-68046-7

Ranc, N., Moorcroft, P. R., Ossi, F., \& Cagnacci, F. (2020). Experimental evidence of memory-based foraging decisions in a large wild mammal. BioRxiv. https://doi.org/10.1101/2020.05.23.112912

Riotte-Lambert, L., Benhamou, S., \& Chamaillé-Jammes, S. (2015). How memory-based movement leads to nonterritorial spatial segregation. The American Naturalist, 185(4), E103-E116. https://doi. org/10.1086/680009

Schlägel, U. E., \& Lewis, M. A. (2014). Detecting effects of spatial memory and dynamic information on animal movement decisions. Methods in Ecology and Evolution, 5, 1236-1246. https://doi. org/10.1111/2041-210X.12284

Spencer, W. D. (2012). Home ranges and the value of spatial information. Journal of Mammalogy, 93(4), 929-947. https://doi.org/ 10.1644/12-MAMM-S-061.1

Van Moorter, B., Visscher, D., Benhamou, S., Börger, L., Boyce, M. S., \& Gaillard, J.-M. (2009). Memory keeps you at home: A mechanistic model for home range emergence. Oikos, 118(5), 641-652. https:// doi.org/10.1111/j.1600-0706.2008.17003.x

How to cite this article: Ranc N, Cagnacci F, Moorcroft PR.

Knowing your neighbours: How memory-mediated conspecific avoidance influences home ranges. J Anim Ecol. 2020;89:27462749. https://doi.org/10.1111/1365-2656.13374 